\title{
Liquid crystal experiment for undergraduate engineering students
}

\section{Mansoor Jalil}

Mansoor B. A. Jalil, "Liquid crystal experiment for undergraduate engineering students," Proc. SPIE 9663, Eighth International Topical Meeting on Education and Training in Optics and Photonics, 96632I (6 October 2003); doi:

$10.1117 / 12.2207469$

EDent: Eighth International Topical Meeting on Education and Training in Optics and Photonics, 2003, Tucson, Arizona, United States 


\title{
Liquid crystal experiment for undergraduate engineering students
}

\author{
Mansoor B. A. Jalil \\ Department of Electrical and Computer Engineering, National University of Singapore, \\ 4 Engineering Drive 3, Singapore 117576 \\ elembaj@nus.edu.sg
}

\begin{abstract}
An undergraduate experiment to investigate the optical properties of liquid crystals (LC) is described. The target students are final-year undergraduates pursuing an advanced optoelectronics course at the Electrical and Computer Engineering Department of the National University of Singapore. In the first part of the experiment, the retardation of light by a LC cell is determined by analyzing the polarization state of light passing through the cell. In the second part, the time response of the LC molecules is obtained by applying a time-varying voltage to the LC cell. The objectives of the experiment are to i) provide a hands-on experience of an optical source-modulator-detector system, in response to the needs of industry, ii) illustrate a practical application of Jones matrix analysis, iii) establish a link between the molecular properties of an LC medium and their optical properties and iv) show how the material properties of a LC material may influence its use in display devices.

(C) 2003 Optical Society of America

OCIS codes: (160.3710) Liquid crystals; (230.2090) Electro-optical devices; (260.1440) Birefringence
\end{abstract}

\section{Introduction}

An advanced course on optoelectronics [1] has been taught to final-year undergraduates at the Electrical and Computer Engineering Department of the National University of Singapore for 15 years. The course is designed to enable students to acquire understanding of the fundamental principles underlying device operation, as well as the technological knowledge relevant to the photonics-related industry. The course contents are constantly updated based on developments in the photonics technology.

Broadly, the course covers the basic physics of light emission, modulation and detection, in semiconductors, electro-optic crystals and liquid crystals (LC). In recent years, greater emphasis has been placed on the study of LC. This trend is in tandem with the growth of the LCD (liquid crystal display) manufacturing activity in Singapore, as highlighted by the recent investment by Toshiba and Matsushita, worth USD1.1 billion, to produce advanced poly-Si thin-film transistor LCDs in Singapore [2]. The course lectures on LC adopt a multi-disciplinary approach in presenting this large and rapidly developing field. They cover (a) a brief introduction on the basic structural and chemical properties of LCs; (b) the physics of light interaction with the electric dipole of the LC molecule (resulting in birefringence) and of the elastic, viscous and electric forces [which give rise to the twisted nematic (TN) configuration]; (c) the operation of a TN cell which is a key component of a LCD and (d) the large-scale integration and addressing of these cells in a LCD.

The class experiment described in this paper provides the necessary practical complement to the lecture materials. Through the experiment, students will investigate how a monochromatic light beam is modulated a LC material, and how to characterize this modulation using an optical detection system. The results of the experiment will re-emphasize the theory concerning the birefringence property of LCD which is central to the TN cell operation. The student will also study the time response of the LC molecules and relate this to the forces holding the LC molecules together.

\section{Overview of Liquid Crystals}

Although liquid crystals were discovered in 1888 by Renitzer, its use in electronically addressed displays dates back to the 1960's only. A breakthrough for LC applications occurs with the discovery of MBBA in 
the late 1960's [3], which exhibits the nematic phase at room temperature. This was followed by the discovery of cyanobiphenyls [4], which are more stable to heat and light and have strong birefringence property, thus paving the way for LC display applications. On the technology side, the development of the TN cell in 1967 resulted in LC readout displays in calculators, watches and other devices. Since then intensive research in academia and industry has resulted in the development of new types of LC materials such as super-twisted nematic and polymer-dispersed LC materials, with enhanced properties. Running parallel to this materials research is the rapid progress in LCD technology, such as the development of poly-Si thin-film transistors (TFT). These advances have led to a dramatic fall in the price of LC flat-panel displays. It is estimated that by the year 2005, flat panel displays, of which LCD forms a major proportion, will replace conventional cathode-ray tube (CRT) displays in personal computers and televisions. There is also an increasing demand for small LCDs in PDAs, mobile phones, digital cameras and other hand-held devices.

\section{Objectives of Experiment}

It is thus important to impart a practical knowledge of a basic LC modulator system to students in the optoelectronics course. For this purpose, a class experiment based on a LC system is designed.

The objectives of the experiment:

i) provide a hands-on experience of an optical source-LC modulator-detector system,

ii) provide a practical application of Jones matrix,

iii) establish a link between the molecular and optical properties of an LC medium,

iv) demonstrate external bias control of the LC optical properties - the basis of display applications.

\section{Description of Experimental System}

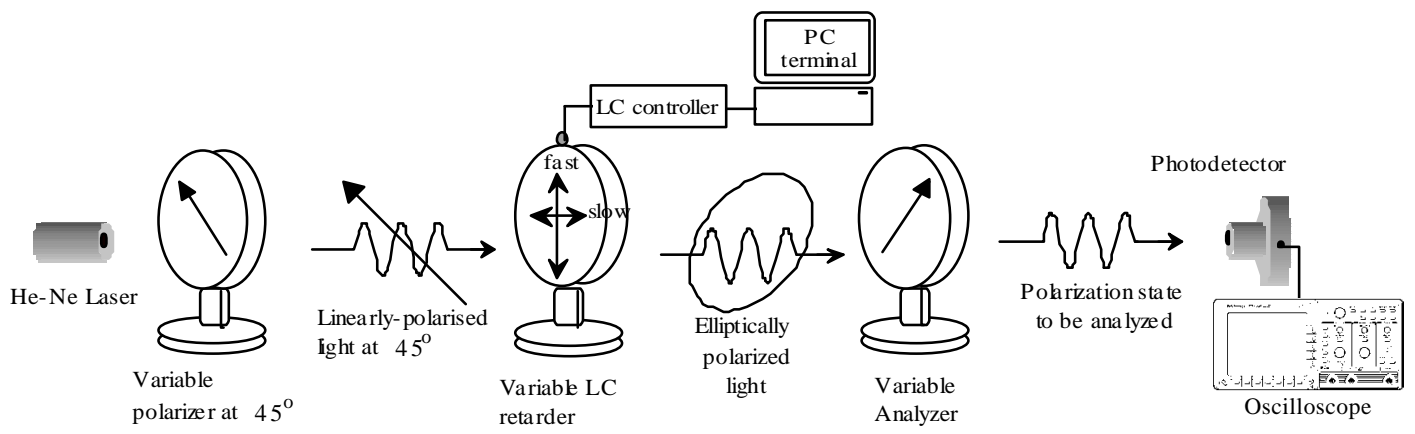

Fig. 1. Schematic diagram of optical set-up.

A photograph of the optical part of the set-up is shown in Fig. 1(a). The full experimental set-up consists of

i) Source: He-Ne Laser with monochromatic output at $\lambda=632.8 \mathrm{~nm}$.

ii) Modulators: Polarizer, Analyzer and $N 4$ Waveplate

iii) LC (Retarder) Cell

iv) LC Controller Interface Card

v) Software for LC Controller Card

vi) Detector: Si Photodetector

vii) Digital Oscilloscope

viii) Vibration-free Optical Table

The source, modulator components, LC cell and photodetector are fixed on the optical table such that they lie along a single axis. The digital oscilloscope are connected to both the photodetector and the LC controller card, and displays both the voltage applied to the LC cell (input) and the intensity of modulated light (output) - see Fig. 1(b). The controller card and controller software enables the student to generate a periodic square wave voltage of variable amplitude across the LC cell (see Section 8). 

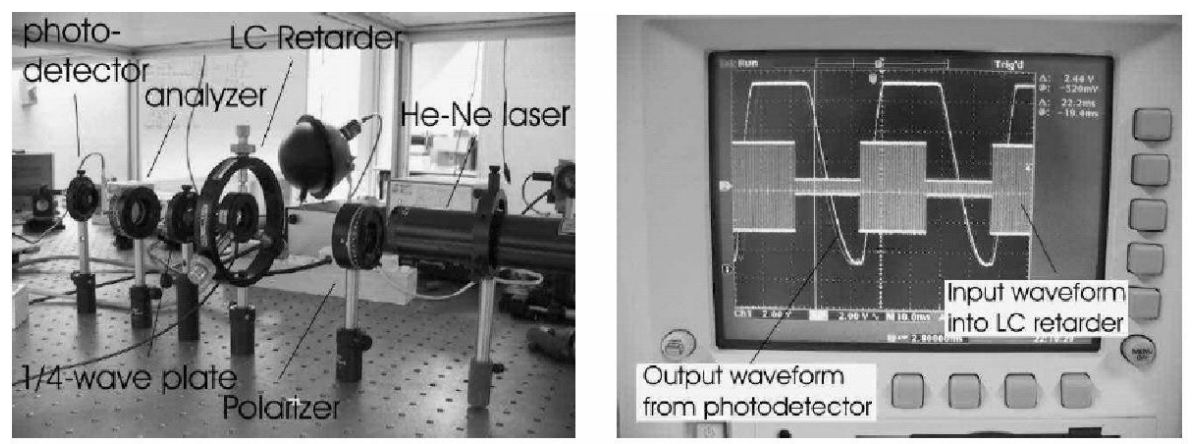

Fig. 2. (a) Photograph of optical components and (b) oscilloscope output showing input bas (square-profile) applied to LC cell and photodetector output (curve).

\section{Investigating the polarization state of light}

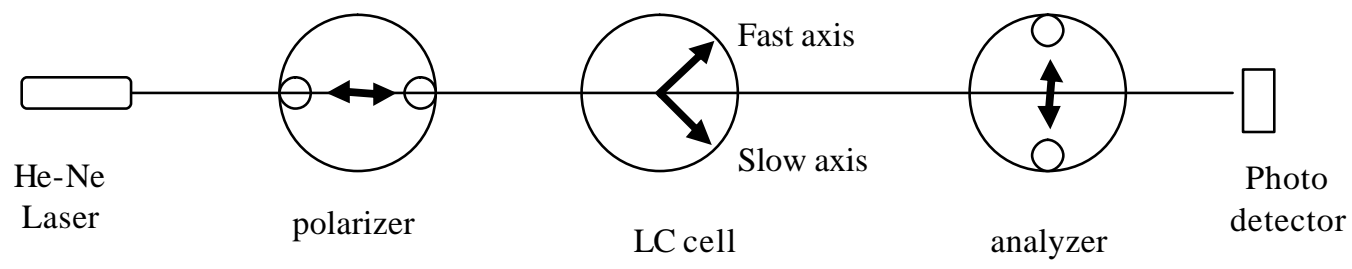

Fig. 3. Schematic diagram of axes (bold arrows) of optical components.

\section{Method:}

The students are to set up the optical components and to pass the He-Ne laser light through the LC cell, sandwiched between the polarizer-analyzer pair. The polarizer axis is aligned at $45^{\circ}$ to the fast axis of the LC cell (as indicated by the bold arrows in Fig. 3). An a.c. voltage of constant amplitude $5 \mathrm{~V}$ at $2 \mathrm{kHz}$ frequency is applied to the LC cell. The angular dependence of the transmitted light is obtained by rotating the analyzer axis and recording the photodetector output at $10^{\circ}$ intervals.

\section{Theory:}

Aligning the polarizer at $45^{\circ}$ to the LC fast axis will ensure that the incident light beam is equally divided into two components, polarized respectively along the fast and slow axes of the LC cell. The LC cell exhibits birefringence, due to the anisotropic nature of the LC molecules. Thus, light polarized along the cell's fast axis will experience a smaller refractive index compared to light polarized perpendicular to that axis. This results in a phase difference (or retardation) $\phi$ between the two polarizations. Depending on the value of $\phi$, light emerging out of the cell will be elliptically polarized to different degrees (full circular polarization if $\phi=\pi / 2+n \pi$, and linearly polarized if $\phi=n \pi$, where $n$ is an integer).

A mathematical representation of an elliptically polarized light is straightforward, i.e. $(1, \exp (\phi))^{T}$ in Jones matrix form. However, it is difficult to visualize in real life the characteristics of an elliptically polarized light. One significant difference between elliptically and linearly polarized light is that only the former does not undergo complete extinction at any point in the rotation $\theta$ of the analyzer axis, as shown in the sample experimental plot of Fig. 4(a). 


\section{Sample Results:}
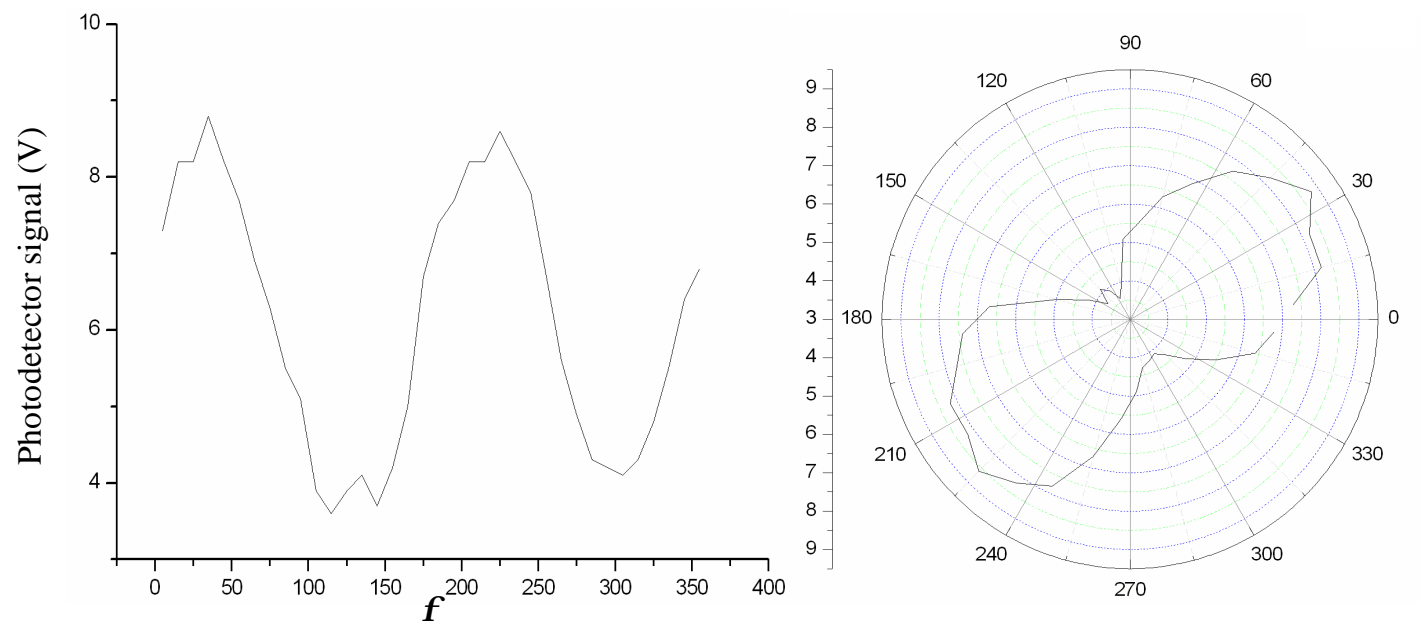

Fig. 4(a) Angular ( $\phi$ ) dependence of transmitted intensity. (b) Polar plot of graph (a).

Analysis:

Jones matrix analysis is used to explain the observed results. First, consider the Jones vector for the elliptically polarized light $\left(\mathbf{v}_{1}\right)$ formed by passing a linearly polarized light at $45^{\circ}\left(\mathbf{v}_{0}\right)$ through the LC cell $\left(\mathbf{M}_{1}\right)$, which introduces a retardation of $\phi$ :

$$
\mathbf{v}_{1}(\phi)=\mathbf{M}_{1}(\phi) \cdot \mathbf{v}_{0}=\left(\begin{array}{cc}
1 & 0 \\
0 & \exp (i \phi)
\end{array}\right) \cdot\left(\begin{array}{l}
1 \\
1
\end{array}\right)=\left(\begin{array}{c}
1 \\
\exp (i \phi)
\end{array}\right)
$$

(a)

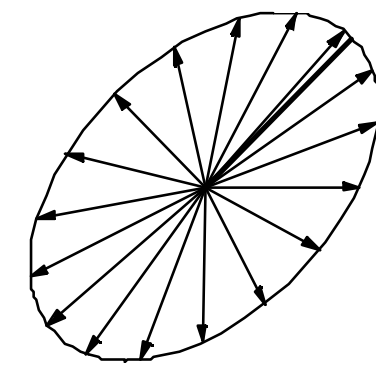

(b)

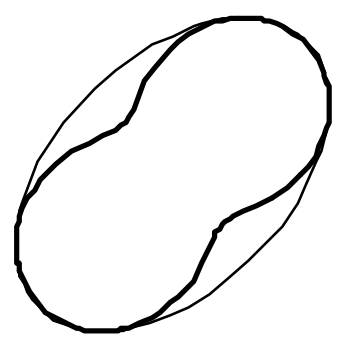

Fig. 5. (a) shows an elliptically polarized light represented by $\mathbf{v}_{1}$ in Eq. (1). The $\mathbf{E}$-field vector traces out an ellipse with a major/minor axes at $\pm 45^{\circ}$ (bold line). (b) Polar plot of the calculated transmitted intensity. [Eq. (6)]. The enclosing ellipse is the polarization ellipse of the detected light.

To find the transmitted intensity through a rotated analyzer, one needs to obtain the Jones matrix for an analyzer oriented at an arbitrary angle $\theta$. For an analyzer along the $x$-axis, the Jones matrix is

$$
\mathbf{A}(0)=\left(\begin{array}{ll}
1 & 0 \\
0 & 0
\end{array}\right)
$$

For an analyzer at an arbitrary angle $\theta$, one has to perform a transformation using the rotation operator:

$$
\mathbf{R}(\theta)=\left(\begin{array}{cc}
\cos \theta & \sin \theta \\
-\sin \theta & \cos \theta
\end{array}\right)
$$


Thus, after transformation, the Jones matrix for the rotated analyzer is given by

$$
\mathbf{A}(\theta)=\mathbf{R}^{T}(\theta) \cdot \mathbf{A}(0) \cdot \mathbf{R}(\theta)=\left(\begin{array}{cc}
\cos ^{2} \theta & \cos \theta \sin \theta \\
\cos \theta \sin \theta & \sin ^{2} \theta
\end{array}\right) .
$$

The transmitted light $\mathbf{v}_{2}$ after passing through the analyzer is then

$$
\mathbf{v}_{2}(\phi)=\mathbf{A}(\theta) \cdot \mathbf{v}_{1}(\phi)=\left(\begin{array}{cc}
\cos ^{2} \theta & \cos \theta \sin \theta \\
\cos \theta \sin \theta & \sin ^{2} \theta
\end{array}\right) \cdot\left(\begin{array}{c}
1 \\
\exp (i \phi)
\end{array}\right) \equiv\left(\begin{array}{c}
v_{x}^{\prime}(\phi, \theta) \\
v_{y}^{\prime}(\phi, \theta)
\end{array}\right) .
$$

The photodetector detects a time-averaged signal of the transmitted light, i.e.

$$
\begin{gathered}
\left\langle I_{\text {detected }}\right\rangle=\left\langle\operatorname{Re}\left[v_{x}^{\prime}(\phi, \theta) e^{i \omega t}\right]^{2}+\operatorname{Re}\left[v_{y}^{\prime}(\phi, \theta) e^{i \omega t}\right]^{2}\right\rangle \\
=\frac{\omega}{2 \pi} \int_{0}^{2 \pi / \omega} \operatorname{Re}\left[v_{x}^{\prime}(\phi, \theta) e^{i \omega t}\right]^{2}+\operatorname{Re}\left[v_{y}^{\prime}(\phi, \theta) e^{i \omega t}\right]^{2} d t \\
=\frac{1}{2}\left[(\cos \theta+\sin \theta \cos \phi)^{2}+\sin ^{2} \theta \sin ^{2} \phi\right] .
\end{gathered}
$$

The transmitted intensity and the corresponding polarization ellipse are plotted together in Fig. 5(b). The plot qualitatively agrees with the experimentally observed dumbbell-shaped variation as shown in Fig. 4. Additionally, both the calculated and experimental plots have their major axis at $45^{\circ}$. It is, however, not possible to quantitatively determine the retardation $\phi$ directly from this angular dependence.

\section{Determining the phase retardation due to $\mathrm{LC}$ cell}

In this section, the student will perform two methods to quantitatively determine the phase retardation caused by the LC cell.

\section{Method 1:}

The same set-up as in Section 5 is used. The only difference is that the analyzer is fixed at $90^{\circ}$ to the polarizer, and that a variable voltage $V$ is applied to the $\mathrm{LC}$ from 0 to $10 \mathrm{~V}$. The intensity of the detected light is then plotted against $V$. A sample plot is shown in Fig. 6(a).

\section{Analysis}

As before, we first obtain the Jones vector for the transmitted light. We take the $x$ and $y$ axes to coincide with the polarizer and analyzer axes, respectively. Then, the LC cell, whose axis is oriented at $45^{\circ}$ to the $x$ axis, is represented by the Jones matrix:

$$
\begin{gathered}
\mathbf{M}_{2}(\phi)=\mathbf{R}(-\pi / 4) \cdot \mathbf{M}_{1}(\phi) \cdot \mathbf{R}(\pi / 4) \\
=\frac{1}{\sqrt{2}}\left(\begin{array}{cc}
1 & -1 \\
1 & 1
\end{array}\right) \cdot\left(\begin{array}{cc}
1 & 0 \\
0 & \exp (i \phi)
\end{array}\right) \cdot \frac{1}{\sqrt{2}}\left(\begin{array}{cc}
1 & 1 \\
-1 & 1
\end{array}\right) \\
=\frac{1}{2}\left(\begin{array}{cc}
1+e^{i \phi} & 1-e^{i \phi} \\
1-e^{i \phi} & 1+e^{i \phi}
\end{array}\right) .
\end{gathered}
$$

Thus, the Jones vector of light after passing through the analyzer $\mathbf{w}_{2}$ is ( $\mathbf{w}_{1}$ denotes the $x$-polarized light due to the polarizer): 


$$
\begin{gathered}
\mathbf{w}_{2}=\mathbf{A}(\pi / 2) \cdot \mathbf{M}_{2}(\phi) \cdot \mathbf{w}_{1} \\
=\left(\begin{array}{ll}
0 & 0 \\
0 & 1
\end{array}\right) \cdot \frac{1}{2}\left(\begin{array}{cc}
1+e^{i \phi} & 1-e^{i \phi} \\
1-e^{i \phi} & 1+e^{i \phi}
\end{array}\right) \cdot\left(\begin{array}{l}
1 \\
0
\end{array}\right)=\frac{1}{2}\left(\begin{array}{c}
0 \\
1-e^{-i \phi}
\end{array}\right)
\end{gathered}
$$

The corresponding $\mathbf{E}$ field is then given by

$$
\mathbf{E}=\frac{E_{0}}{2}\left(1-e^{i \phi}\right) e^{i \omega t} \hat{\mathbf{j}}
$$

and the intensity is then

$$
\begin{gathered}
I=|E|^{2}=E \times E^{*}=\frac{E_{0}}{2}\left(1-e^{i \phi}\right) e^{i \omega t} \times \frac{E_{0}}{2}\left(1-e^{-i \phi}\right) e^{-i \omega t}=\frac{E_{0}^{2}}{2}(1-\cos \phi) \\
\Rightarrow I=\frac{I_{\max }}{2}(1-\cos \phi) .
\end{gathered}
$$

The retardation is then given [taking into account the $2 \pi$-periodicity with retardation $\phi$, as can be seen in Fig. 6(a)]:

$$
\phi=\cos ^{-1}\left(1-\frac{2 I}{I_{\max }}\right)+2 n \pi .
$$

To determine the value of $n$ at a given voltage $V$, one notes that retardation $\phi$ and hence $n$ is smallest at the largest $V$. Thus, by referring to Fig. 6(a), we find, for example, that for $1.72<V<10, n$ has a value of 0 , while for $1.16<V<1.72, n=1$, and so on.

To obtain the retardation $\phi_{0}$ at zero voltage, we note that $I(0)=3.0 \mathrm{~V}$, while $I_{\max }=10.8 \mathrm{~V}$. There are a total of 3 oscillations, i.e. $n=3$ at $V=0$. Thus

$$
\phi_{0}=\cos ^{-1}\left(1-\frac{2 \times 3.0}{10.7}\right)+2 \times 3 \pi=19.6 .
$$

From Eq. (10), the transmitted intensity $I$ can be converted to retardation $\phi$ and then plotted against voltage [Fig. 6(b)].
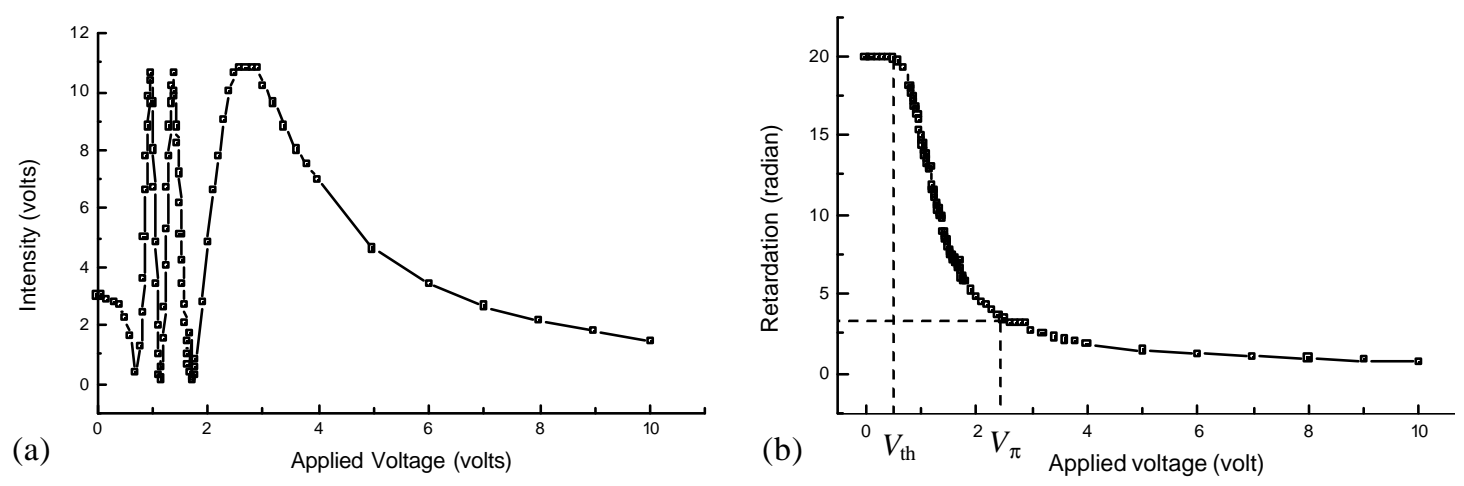

Fig. 6. (a) Plot of experimentally measured transmitted intensity vs. $V$. (b) Plot of retardation $\phi$ vs. $V$.

From this plot, we can determine two important quantities of an LC retarder, i.e. its threshold voltage $V_{\text {th }}$ and the half-voltage $V_{\pi} . V_{\text {th }}$ is the voltage at which the retardation starts to decrease (when the LC molecules start to align themselves with the applied electric field). $V_{\pi}$ is the voltage at which there is a phase shift of $\pi$ (antiphase). From Fig. 6(b), we obtain $V_{\pi}=2.3 \mathrm{~V}$, and $V_{\mathrm{th}}=0.45 \mathrm{~V}$. 
Method 2:

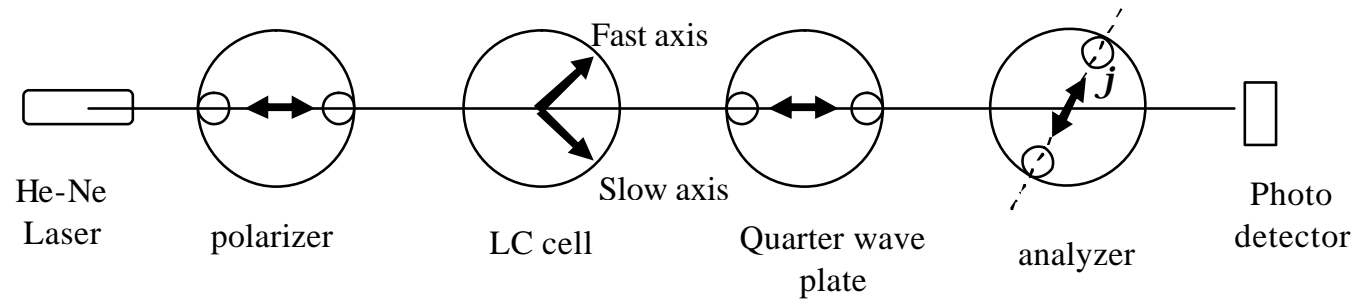

Fig. 7. Schematic diagram of axes (bold arrows) of optical components.

A cleaner method to determine the retardation $\phi$ is by placing a $\lambda 4$ waveplate at $45^{\circ}$ to the LC cell (as shown above). In this configuration, the $\mathrm{LC}$ cell acts as a voltage-modulated polarization rotator. A voltage of $2 \mathrm{~V}$ at $2 \mathrm{kHz}$ is applied on the LC cell and the analyzer axis is rotated. The axis orientation $\varphi$ relative is recorded where maximum transmission occurs. This is repeated for an applied voltage range of $2 \mathrm{~V}<V<10 \mathrm{~V}$, and the variation of $\varphi$ plotted against $V$. The sample results are plotted in Fig. 8.

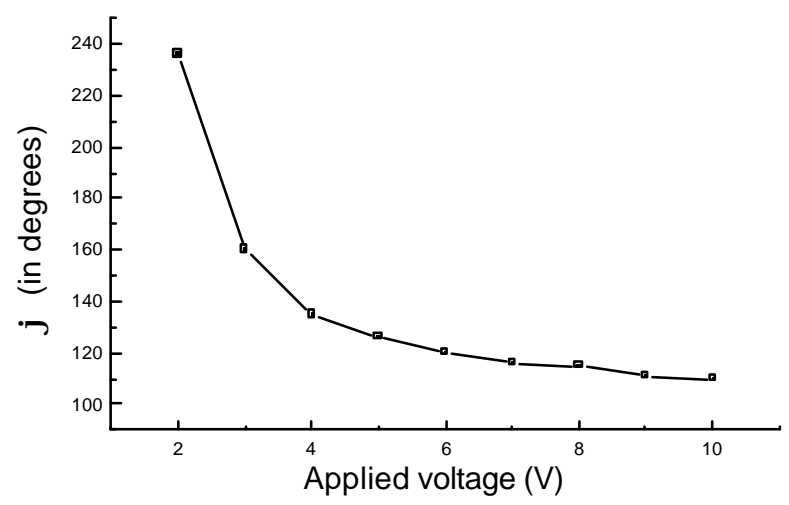

Fig. 8. Plot of analyzer orientation $\phi$ at maximum transmission vs. $V$.

Analysis

The $\lambda 4$ plate converts the elliptically polarized light emerging out of the LC cell into a linearly-polarized one. The orientation of the plane of polarization $\varphi$ w.r.t. to the $\lambda 4$ plate axis is proportional to the retardation $\phi$. Thus, the above graph has the same shape as the high voltage range of Fig. 6(b). In fact, $\varphi=\phi / 2$, as can be seen in the following Jones matrix analysis:

Let $\mathbf{u}_{1}$ be the Jones vector of light emerging from the LC. From earlier analysis, we have

$$
\begin{aligned}
\mathbf{u}_{1} & =\mathbf{M}_{2}(\phi) \cdot \mathbf{w}_{1}=\frac{1}{2}\left(\begin{array}{cc}
1+e^{i \phi} & 1-e^{i \phi} \\
1-e^{i \phi} & 1+e^{i \phi}
\end{array}\right) \cdot\left(\begin{array}{l}
1 \\
0
\end{array}\right)=\frac{1}{2}\left(\begin{array}{c}
1+e^{i \phi} \\
1-e^{i \phi}
\end{array}\right) \\
& =\frac{e^{i \phi / 2}}{2}\left(\begin{array}{l}
e^{-i \phi / 2}+e^{i \phi / 2} \\
e^{-i \phi / 2}-e^{i \phi / 2}
\end{array}\right) \equiv\left(\begin{array}{c}
\cos (\phi / 2) \\
-i \sin (\phi / 2)
\end{array}\right),
\end{aligned}
$$

after disregarding a phase factor. When $\mathbf{u}_{1}$ passes through the $\lambda 4$ plate (Jones matrix $\mathbf{Q}$ ), we have

$$
\mathbf{u}_{2}=\mathbf{Q} \cdot \mathbf{u}_{1}=\left(\begin{array}{cc}
1 & 0 \\
0 & -i
\end{array}\right) \cdot\left(\begin{array}{c}
\cos (\phi / 2) \\
-i \sin (\phi / 2)
\end{array}\right)=\left(\begin{array}{c}
\cos (\phi / 2) \\
-\sin (\phi / 2)
\end{array}\right),
$$

which is a linearly polarized light oriented at $\phi / 2$ to the $x$-axis. This provides proof that the LC cell and $\lambda 4$ plate combination acts as a polarization rotator. 


\section{Determining the orientation of LC molecules}

The aim of this section is to relate the experimentally measured retardation of light to the LC microscopic property (i.e. angular tilt $\psi$ ) of the LC molecules. The retardation is related to the anisotropy of the LC refractive, i.e.

$$
\phi=\frac{2 \pi\left(n(\psi)-n_{0}\right) d}{\lambda}
$$

where $d$ is the cell thickness, $n_{\mathrm{o}}$ is the ordinary refractive index (experienced by light polarized along the LC cell fast axis), and $n(\psi)$ is the refractive index for polarization along the slow axis at tilt angle $\psi$. The tilt dependence of $n(\psi)$ is given by:

$$
\frac{1}{n^{2}(\psi)}=\frac{\cos ^{2} \psi}{n_{\mathrm{e}}^{2}}+\frac{\sin ^{2} \psi}{n_{\mathrm{o}}^{2}},
$$

where $n_{\mathrm{e}}$ is the extraordinary index of the LC material. From the LC cell specifications, we have $d=8.38 \times 10^{-6} \mathrm{~m}, n_{\mathrm{o}}=1.5103$ and $n_{\mathrm{e}}=1.7445$.

Note that at $V=10 \mathrm{~V}$, the LC molecules are more or less fully aligned along the electric field, i.e. $\psi=90^{\circ}$. The LC cell is thus optically inactive, with $n(\psi)=n_{\mathrm{o}}$ and $\phi=0$. In practice, however, we obtain some residual retardation $\phi_{\mathrm{offset}}$ due to axis misalignment of optical components, etc. This offset has to be subtracted in order to obtain the actual retardation due to the birefringence of the LC material.

Inverting Eq. (16), we have

$$
\phi=\cos ^{-1}\left(\sqrt{\frac{\frac{1}{n^{2}(\psi)}-\frac{1}{n_{\mathrm{o}}^{2}}}{\frac{1}{n_{\mathrm{e}}^{2}}-\frac{1}{n_{\mathrm{o}}^{2}}}}\right) .
$$

Thus, from Eqs. (15) and (17), we can use the data obtained in Section 6 to plot the angular tilt $\psi$ as a function of the applied voltage $V$ [Fig. 9(a)].
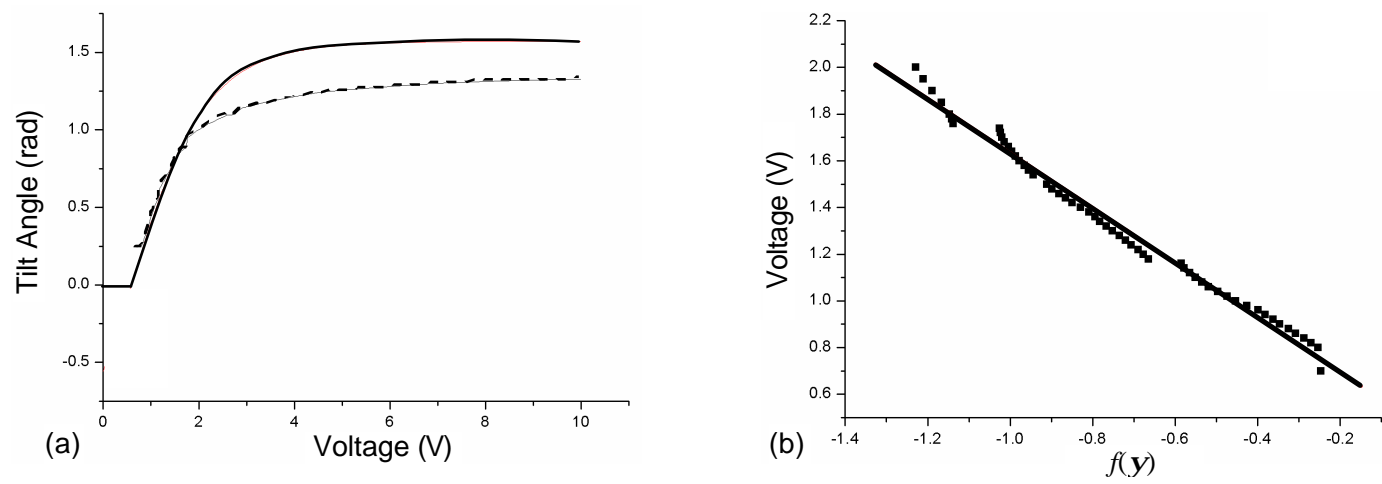

Fig. 9. (a) Plot of tilt angle $\psi$ vs. $V$. Dotted line denotes experimental data, while solid line is the theoretical prediction. Linear plot obtained from (a) after some algebraic manipulation.

The solid line in Fig. 9(a) from the theoretical expression relating $\psi$ to $V$, i.e. [5] 


$$
\psi= \begin{cases}0 & V<V_{t h} \\ \frac{\pi}{2}-2 \tan ^{-1}\left[\exp \left(-\frac{V-V_{t h}}{V_{0}}\right)\right] & V>V_{t h}\end{cases}
$$

We can rearrange the above equation, so as to obtain a suitable linear curve to plot and determine the value of $V_{0}$, which is a measure of the "rigidity" of the LC molecules. From Eq. (18) above, we obtain

$$
V=V_{\text {th }}-V_{0} \ln \left\{\tan \left[\frac{1}{2}\left(\frac{\pi}{2}-\psi\right)\right]\right\} \equiv V_{\text {th }}-V_{0} f(\psi),
$$

$V$ is plotted against $f(\psi)$ for the voltage range $V_{\text {th }}<V<2.0$ [Fig. 9(b)], and $V_{0}$ determined from the gradient. It was found that $V_{0}=0.994 \mathrm{~V}$.

\section{Time response of LC molecules}

In this section, we investigate a property of LC molecules which is a major shortcoming as far as dynamic (video) applications are concerned, i.e. their slow time response $\tau$. For normal TN LCs, $\tau$ are in the range of several milliseconds.
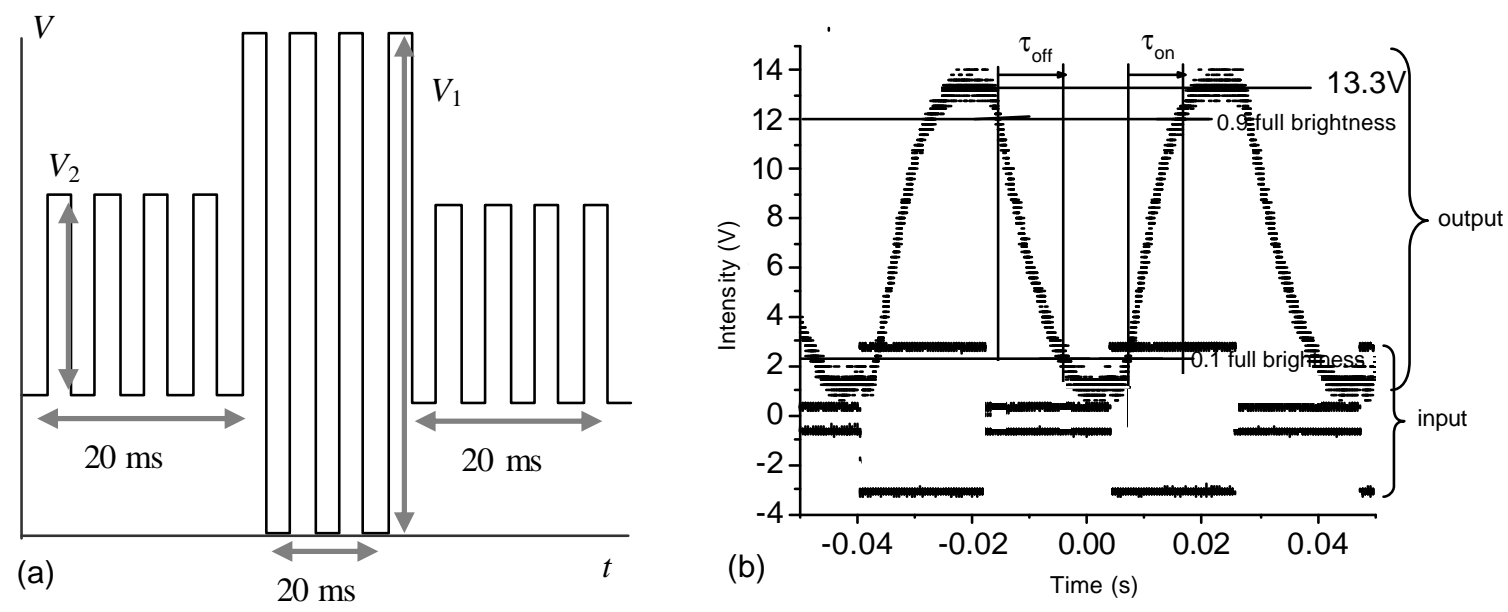

Fig. 10. (a) Time-dependent voltage profile applied to LC cell. (b) Sample output printed from oscilloscope.

\section{Method:}

We use the same set-up as Section 5, i.e. with the $\lambda 4$ plate removed. Next, using the controller software, such that an AC bias signal consisting of two amplitudes $V_{1}$ and $V_{2}$, is applied to the LC cell via the controller card. The larger amplitude $V_{1}$ corresponds to the final peak in the transmitted intensity $I$ of Fig. 7 $(\sim 3 \mathrm{~V})$, while $V_{2}$ is set at a sub-threshold value of $0.5 \mathrm{~V}$. The time duration for both $V_{1}$ and $V_{2}$ is set at $\sim 20$ ms. The signal from the LC controller is fed into Channel 1, while the photodetector output to Channel 2 of the oscilloscope. Both channels are displayed simultaneously [Fig. 10(b)]. From the output profile, $\tau_{\text {on }}$, the rise time to $90 \%$ of full brightness, and $\tau_{\text {off }}$, the decay time from maximum to $10 \%$ of full brightness are obtained.

Analysis

From the sample output: $\tau_{\mathrm{on}}=-0.004042-(-0.01568) \mathrm{s}=11.64 \mathrm{~ms}$

$$
\tau_{\text {off }}=0.01677-0.007252 \mathrm{~s}=9.52 \mathrm{~ms}
$$


Theoretically, the rise time constant $\tau_{\mathrm{on}}$ is dependent on several to the material properties of the LC material [6]

$$
\tau_{o n}=\frac{\gamma d^{2}}{k \pi^{2}}\left[\left(\frac{V}{V_{t h}}\right)^{2}-1\right]^{-1}
$$

where $\gamma$ and $k$ are the rotational viscosity and elastic constants of the LC molecules. In addition, the threshold voltage $V_{t h}$ is also related to the material properties as follows:

$$
V_{t h}=\frac{k \pi^{2}}{\Delta \varepsilon},
$$

where $\Delta \varepsilon$ is the anisotropy in permittivity.

$$
\Delta \varepsilon=\Delta\left(n^{2}\right)=\left(n_{\mathrm{e}}^{2}-n_{\mathrm{o}}^{2}\right)=\left(1.7445^{2}-1.5103^{2}\right)=0.7623 .
$$

Substituting the numerical values into Eqs. (20) and (21), the rotational viscosity constant is found to be $\gamma=2.00 \times 10^{6} \mathrm{Ns} \mathrm{m}^{-2}$.

\section{Conclusions}

On completion of the experiment, the student will gain experience in setting up an optical measurement system, and be familiar with various optical components and associated electronics to control/display the system inputs and outputs. A high degree of mathematical analysis of the experimental data is required. This ensures that students attain proficiency of the mathematical tools used in optics (principally that of Jones analysis), and thus gain rigorous understanding of the optical properties of LC material. Students will also discover the relationship between microscopic properties (molecular orientation, elastic forces) and macroscopic quantities (birefringence, time response). More importantly, they will learn that the LC optical properties can be systematically controlled by external biases. This external control forms the basis of LC application in displays and other devices.

\section{Miscellaneous}

The author wishes to acknowledge Ms. M. Hussain, Mr. X. Wang, Miss M. H.-F. Lim, Mr. G. K. Chowdhury and Miss P. Wadhwa for their assistance in the experiment, and the Electrical and Computer Engineering Department, National University of Singapore for sponsoring this conference presentation.

\section{References}

[1] S. J. Chua and M. B. A. Jalil, "Optoelectronics for electrical and computer engineering students," in The Seventh International Conference on Education and Training in Optics and Photonics, T-K. Lim and A. H. Guenther, eds., Vol. 4588 of OSA Proceedings Series (Optical Society of America, Washington, D.C., 2002), pp. 182-191.

[2] Toshiba and Matsushita Agree to Establish a Joint Venture For Manufacturing LCD Panels in Singapore, http://www.toshiba.co.jp/about/press/2001 02/pr2301.htm.

[3] H. Kelker, B. Scheurle, H. Hatz, and W. Bartsch, "Liquid crystalline phase having particulary low freezing points," Angew. Chem. Int. Ed. 9, 962-963 (1970).

[4] G. W. Gray, K. J. Harrison, and J. A. Nash, "New family of nematic liquid crystals for displays,” Electron. Lett. 9, 130-131 (1973).

[5] See e.g. P.-G. de Gennes, The Physics of Liquid Crystals, (Clarendon Press, Oxford, 1974), Chap. 3.

[6] T. Tsukada, TFT/LCD Liquid-Crystal Displays Addressed by Thin-Film Transistors, (Gordon and Breach Publishers, Singapore, 1996), Chap. 5. 